across all three cohorts. Organisational culture and readiness for EBP in the selected Schools of Nursing/Midwifery, and in the selected hospitals, was found to be moderate, but with a lot more to be done.

Conclusions The positive beliefs in EBP, and moderate organisational culture/readiness for EBP are encouraging findings. Nurses/midwives in academic and clinical settings are positively predisposed to EBP and believe their workplaces as moderately supportive of its implementation. However, the very low EBP implementation levels are concerning, both for nurse/midwifery education and practice going forward, and particularly for patient care and safety. Close scrutiny of the quantitative data reveals a substantial deficit in knowledge and understanding of EBP among nurses and midwives, and this is clearly corroborated by the qualitative data generated by the open question. These findings offer an informed starting point from which a specifically tailored education programme, underpinned with knowledge of the specific needs of nurses/midwives, as well as challenges and opportunities in their workplaces, can be developed and implemented with the aim of developing/improving EBP knowledge and skills to foster a culture of EBP.

\section{INTEGRATED INTERVENTIONS TO REDUCE PRESSURE ON ACUTE HOSPITALS: A SYSTEMATIC UMBRELLA REVIEW}

Martin Keane, Camille Coyle, Louise Farragher, Gerald O'Nolan, Aoife Cannon, Jean Long. Health Research Board, Dublin, Ireland

\subsection{6/bmjebm-2019-EBMLive.32}

Objectives The objectives of this review were to examine integrated health system interventions that have the potential to reduce pressure on acute hospitals. The outcomes that were included for assessing reduced pressure were unplanned admissions to hospital, readmissions, length of stay in hospital, emergency department visits, and healthcare costs.

Method An initial scoping search was conducted in order to frame the parameters of the review. Following the scoping search, an information specialist developed a targeted search strategy using $\mathrm{MeSH}$ terms and keywords for the outcomes of interest. Two databases, MEDLINE and the Cochrane Database of Systematic Reviews, were included in the search. Pairs of authors screened, quality assessed, and extracted data. Heterogeneity prevented pooled analysis of the included reviews. Instead, we extracted the findings related to health system outcomes from each review and described the effectiveness for each intervention by outcome measured and by population, as well as summarising the findings of the reviews for each intervention. We calculated the degree of overlap of primary studies in our included reviews using the corrected covered area measure.

Results This review included 36 published systematic reviews and one umbrella review. Our analysis identified seven complex integrated interventions targeting adults with chronic diseases, eight interventions focusing on medical and surgical conditions among adults, and three interventions for older people. We identified a total of 13 integrated interventions between the hospital and the community aiming to reduce pressure on acute hospitals. Seven interventions focused on people with chronic diseases. Among these, self-management demonstrated good effectiveness, and the other six were moderately effective (discharge management, chronic care model, complex interventions, multidisciplinary teams, hospital at home, and interactive telemedicine). Four interventions targeting acute conditions were moderately effective (discharge management, medication management, hospital at home, and primary care near emergency department), and four emergency department interventions had low effectiveness. Three interventions focusing on older people also had low effectiveness (discharge management, case management, and specialised multidisciplinary rehabilitation for hip fracture).

Conclusions The findings indicate that there are a number of promising interventions that reduce pressure on acute hospitals for people with chronic diseases. There are also some promising interventions that reduce pressure on acute hospitals for people with medical and surgical conditions. There are currently no promising interventions that reduce pressure on acute hospitals for older people. Integrated interventions are multi-component complex interventions, and the interrelationships between these components are rarely described in the literature. Furthermore, the delivery of interventions requires a complex chain of action, delivered in health systems that combine an array of pre-existing interventions and contextual contingencies. These intervention pathways were rarely explored in the studies we reviewed. Therefore, we were unable to identify why these interventions were promising for only some patients under certain conditions. These limitations make it very difficult to translate research on integrated interventions to reduce pressure on acute hospitals into policy and practice.

\section{THE RISK OF IMPRECISE TERMINOLOGY: INCONGRUENT RESULTS OF CLINICAL TRIALS AND INCONGRUENT RECOMMENDATIONS IN CLINICAL GUIDELINES}

${ }^{1}$ Franz Porzsolt, ${ }^{1,2}$ Felicitas Wiedemann, ${ }^{3}$ Karen Schmaling, ${ }^{4}$ Robert Kaplan. ${ }^{1}$ Institute of Clinical Economics, UIm, Germany; ${ }^{2}$ Diakonie-Klinikum, Stuttgart, Germany; ${ }^{3}$ Washington State University, VancouverWA, USA; ${ }^{4}$ Stanford University, Stanford, USA

\subsection{6/bmjebm-2019-EBMLive.33}

Objectives The aim of our study is to describe the congruence in the terminology used to describe both design and outcomes of clinical trials. Terminology affects the endorsement of commonly accepted clinical experience, and of poorly defined and undifferentiated interpretations of efficacy and effectiveness. Further, choice of terms may explain the results of our congruence analysis of clinical guidelines. A review of recommendations based on 330 scenarios from 24 international guidelines for the same scenario about treatment of malignant diseases found congruence in only $15 \%$ of the corresponding recommendations. ${ }^{1}$

Method We analysed five corresponding pairs of terms recommended in six reviews articles on the reporting the outcomes of clinical trials. ${ }^{2-7}$ These pairs were efficacy/effectiveness outcome, experimental/observational trial, explanatory/pragmatic attitude, randomized/non-randomized allocation of intervention, and analytical/descriptive trial. We also analysed four corresponding triplets of terms used in 100 published quality of life (QoL) studies. The references of these 100 QoL studies were reported previously. ${ }^{8}$ The four triplets of terms were explanatory/pragmatic/not defined attitude, randomized/nonrandomized/not defined allocation of intervention, primary/secondary/not defined endpoint, and efficacy/effectiveness/not defined outcome. References in online version.

Results None of the reviews included all six pairs of terms. The pairs explanatory/pragmatic, and randomized/non- 
randomized were included in 5/6 reviews. ${ }^{2-7}$ The pair efficacy/ effectiveness was - with exceptions ${ }^{2} 5$ - included in four

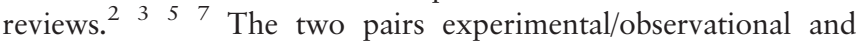
analytical/descriptive were included - with exceptions ${ }^{6}$ - each in two reviews ${ }^{4} 6$ The review by Schwartz \& Lellouch $^{2}$ included the pair of explanatory/pragmatic and discussed efficacy but not effectiveness. The CONSORT statement ${ }^{5}$ included three pairs of terms but did not discuss the meanings of efficacy/effectiveness. The remaining reviews ${ }^{3} 47$ included three but different pairs of terms. Our analysis of the studies confirmed the use of an experimental (RCT) design in 91\%, the assessment of the primary endpoint in $29 \%$, the assessment of efficacy in $14 \%$, of effectiveness in $25 \%$, and neither of these in $60 \%$. In $6 \%$ the study was classified as a pragmatic trial.

Conclusions The most likely reason for the observed incongruence is a lack of a consensus on terms and methods for reporting the results of pragmatic clinical trials. All reviews expect pragmatic trials to describe effects under RWC but assess these effects under experimental but not real-world conditions (RCTs) resulting in a conflict between expected and observed outcomes. CONSORT includes two imprecise statements. ${ }^{5}$ The review by Schwartz \& Lellouch $^{2}$ does not use the term 'randomized' and cannot justify the new name of 'randomized pragmatic trial'.5 Second, the aim of 2008 CONSORT is extension of its applicability to pragmatic trials. In fact, CONSORT changed 'pragmatic' into 'pragmatic randomised' and used an inappropriate reference ${ }^{2}$ to justify this change. The benefit of this maneuver is the recommendation of a valid instrument for assessment of effectiveness, but it may result in harm from the resulting confusion and incongruence of guideline recommendations.

\section{IS THE REVISED COCHRANE RISK OF BIAS TOOL RESEARCH READY FOR THE ERA OF OPEN SCIENCE AND PREREGISTRATION?}

${ }^{1}$ Merlin Bittlinger, ${ }^{2}$ Johannes Schwietering. ${ }^{1}$ Berlin Institute of Health, QUEST - Quality | Ethics | Open Science | Translation, Berlin, Germany; ${ }^{2}$ Hannover Medical School (MHH), Hannover, Germany

\subsection{6/bmjebm-2019-EBMLive.34}

Objectives Risk of bias (RoB) is an important to assess scientific evidence. Lack of detailed preregistration obfuscates reporting transparency and cast doubts about the required bijective relation between study protocol and the final scientific report of outcomes.

This contribution examines whether the lack of preregistration affects the judgments required for risk of bias assessment according to the RoB 2.0 tool and whether the tool can adequatly capture flaws in preregistration.

Method We examined the literature on RoB 2.0 and thoroughly evaluated the definition and criteria for the three different categories 'high risk of bias', 'low risk of bias', and some concerns of risk of bias'. Moreover, we investigated the literature on meta-science and performed a conceptual analysis of the epistemic merits and methodological benefits arising from various forms of preregistration. Accordingly, selective endpoint reporting, or endpoint modification raise serious and severe doubts about the scientific validity of biomedical randomized controlled trials (RCT) and preregistration is obligatory according to Article 35 of the Declaration of Helsinki of 2013.

Results The upcoming revised Cochrane handbook introduces RoB 2.0 as a new research tool for assessing the risk of bias of an RCT. RoB 2.0 requires a comparison between the prespecified analysis intentions and the reported analyses in order to assess potential selection bias of multiple outcomes or endpoints. In case a preregistered analysis plan is met, 'low risk bias' is assigned. 'High risk of bias' is assigned only if it is likely that reported outcomes have been selected based on the results, i.e. a deviation from the preregistered protocol is detected. If no information is available, RoB 2.0 suggests 'some concern'. Furthermore, in cases where preregistration is lacking, RoB 2.0 suggests the methods section of an article as a source of the analysis intentions. Therefore, the lack of preregistration does not by default lead to the evaluation of a 'high risk of bias'.

Conclusions Although lack of preregistration can lead to 'some concerns of risk of bias', there is by default no assignment of 'high risk of bias'even if a preregistration protocol is completely lacking. In light of the epistemic arguments in favour of preregistration, RoB 2.0 presents an untoward loophole in the risk of bias assessment with regard to selective outcome reporting or post hoc endpoint modification. Because any RoB 2.0 assessment is very effort-intensive and time-consuming, it is of utmost importance that all sort of biases are adequate considered and, thus, that future systematic reviews and metaanalysis benefit from risk of bias assessment tools that account for lack of preregistration as a source of 'high risk of bias' by default.

\section{SEX AND GENDER REPORTING AND ANALYSIS IN COCHRANE REVIEWS: A CROSS-SECTIONAL METHODS STUDY. PRELIMINARY RESULTS}

${ }^{1}$ Alba Antequera, ${ }^{1}$ Montserrat León, ${ }^{2}$ Sara Calderón, ${ }^{3}$ Ana Cuadrado. 'Iberoamerican Cochrane Centre - Sant Pau Biomedical Research Institute (IIB Sant Pau), Barcelona, Spain; ${ }^{2}$ Clinical Research Fellow at Queen Mary University of London, London, UK; ${ }^{3}$ London School of Hygiene and Tropical Medicine, London, UK

\subsection{6/bmjebm-2019-EBMLive.35}

Objectives Sex and gender health differences have been well established in the scientific literature. However, numerous studies present poor levels of inclusion of female participants and an insufficient sex/gender reporting and analysis. This lack of consideration of sex and gender in medical research reduces the applicability of findings and jeopardises its capacity to support informed decisions. Studies also suggest that women under-representation in science might be related to this deficient consideration of sex/gender in health research. This study aims to describe the degree of sex/gender reporting and analysis in Cochrane systematic reviews published in 2018 and investigate its association with gender of authorships.

Method Cross sectional study. We screened Cochrane reviews published in 2018. We removed reviews addressing sex-specific medical conditions and those that had been withdrawn by Cochrane. We collected data on gender and country affiliation of first and last authors, and review type. We documented the frequency of sex/gender terms used in each section of the reviews (abstract, methods, results, and discussion). In the 